\title{
Application of a twisted nematic cell in beam steering devices
}

\author{
G. Derfel, ${ }^{* 1}$ M. Buczkowska, ${ }^{2}$ \\ ${ }^{I}$ Institute of Information Technology, Lodz University of Technology, Wólczańska 215, 90-924 Łódź, Poland, \\ ${ }^{2}$ Institute of Physics, Lodz University of Technology, Wólczańska 219, 90-924 Łódź, Poland
}

Received December 16, 2015; accepted December 22, 2015; published December 31, 2015

\begin{abstract}
Several simple beam steering devices composed of twisted nematic cells and typical optical elements are proposed. Principles of operation are described, based on elementary optical phenomena as total internal reflection or polarisation by reflection. The deflection angles are calculated for exemplary parameters. The possibility of modifications of the proposed devices is discussed.
\end{abstract}

Beam steering optical devices find applications wherever the light beam direction needs to be rapidly changed. The devices based on electro-optical effects in liquid crystalline systems are of particular interest because they offer several advantages over mechanical modulators, in particular no need to apply mechanical force to move optical elements, low driving voltage and low cost [1-2]. Nematic liquid crystals are often employed for beam steerers. They are birefringent uniaxial media with positive optical anisotropy, $\Delta n=n_{e}-n_{o}>0$. The orientation of an optic axis can be controlled by means of an external electric field which offers beam steering and other electro-optic applications. Several types of liquid crystal beam steerers can be distinguished, for instance the tunable gratings which employ diffraction of light or the devices in which refraction is used [3-8]. In this paper, we propose several beam steering devices in which the crucial role is played by a typical twisted nematic cell.

The twisted nematic cell (TN) is the most popular liquid crystal device used both in the simplest displays as well as in technologically advanced devices, e.g. in multicolour screens [2]. The nematic layer is confined between two transparent plates with transparent electrodes. When it is not subjected to an external electric field, the optic axis remains parallel to the plates but changes continuously its orientation through $90^{\circ}$ from the first plate to the second plate. If the thickness of the layer is suitably chosen, the plane of polarisation of linearly polarised light, incident normally on one surface of the layer, rotates effectively through $90^{\circ}$. Under the action of voltage significantly exceeding the threshold value $U_{T}$, the twisted structure unwinds. The optic axis becomes perpendicular to the plates and the polarisation of the incident light is not affected. The principle of operation of the devices proposed in the following is based on the

*E-mail: gderfel@p.lodz.pl possibility of switching between two orientations of the polarisation plane of light emerging the twisted nematic cell.

Figure 1 shows the device composed of a TN cell and a Wollaston prism. The Wollaston prism is a kind of polarising beam splitter made of calcite, which is uniaxial birefringent crystal with negative optical anisotropy $\Delta n=n_{e}-n_{o}<0$, where $n_{e}=1.4552$ and $n_{o}=1.6557$ (for $\lambda=632.8 \mathrm{~nm}$ ). The prism is composed of two parts. The optic axis in the first part is perpendicular to the optic axis in the second part. The prism deflects the beam with respect to its initial direction depending on the polarisation of the beam [9].
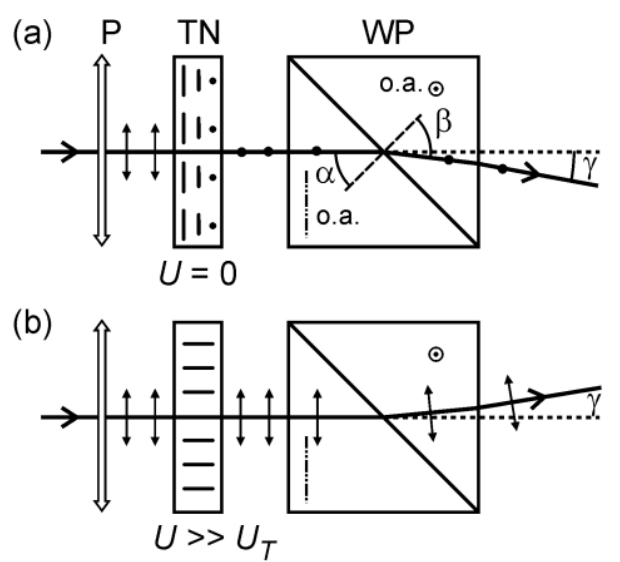

Fig. 1. Twisted nematic cell TN cooperating with a Wollaston prism WP and polariser P. Directions of the optic axes (o.a.) as well as the polarisation of light beams are indicated.

The incident light passes through the polariser $\mathrm{P}$, the twisted nematic cell TN and enters the Wollaston prism WP as shown in Fig. 1. In the absence of bias voltage, the plane of polarisation of light is rotated by $90^{\circ}$ after passing through the twisted structure of the TN cell. In the first half of the prism, the electric field vector of the light wave is perpendicular to the optic axis. The beam behaves as an ordinary ray and has the refractive index $n_{o}=1.6557$. When the ray enters the second part of the prism, the electric field vector is parallel to the optic axis. The ray propagates as the extraordinary ray in this part and the refractive index for it is $n_{e}=1.4552$. Therefore 
refraction away from the normal occurs on the boundary between two parts of the prism. In our example, the prism has the form of a cube. The angle of incidence on the boundary between both parts is $\alpha=45^{\circ}$. The angle of refraction is larger: $\beta=52.02^{\circ}$. The ray emerges the prism after another refraction and makes the angle $\gamma=10.46^{\circ}$ with the initial direction.

If the external voltage significantly exceeding the threshold value is applied to the layer, the structure of the TN cell becomes untwisted. The optic axis of the nematic is parallel to the incident beam. The plane of polarisation is not changed. Therefore the electric field vector of the light wave propagating in the first part of the prism is parallel to the optic axis of calcite. The beam behaves as the extraordinary ray and has the refractive index $n_{e}=1.4552$. In the second part of the prism, the electric field is parallel to the optic axis, therefore the ray changes into an extraordinary ray with the refractive index $n_{o}=1.6557$. The ray is refracted toward the normal. The angle of refraction is now $\beta=39.36^{\circ}$. The ray emerges the prism making the angle $\gamma=9.35^{\circ}$ with the initial direction. The total angle between directions of the rays which can be obtained as a result of application of voltage is $\delta=19.81^{\circ}$.

Another variant of the device composed of TN cells, the Wollaston prism and the analyser $\mathrm{A}$ is presented in Fig. 2. Unpolarised light enters the prism and is split into two rays with mutually perpendicular polarisations. Both rays pass through the two suitably oriented $\mathrm{TN}$ cells steered with the same voltage. Only one ray is transmitted through the analyser as exemplified in Fig. 2. In this way, one can switch between two directions of emerging beams which differ by the angle $\delta=19.81^{\circ}$.

Figure 3 presents the device composed of entrance polariser P, TN cell, the plane-parallel plate GP made of glass with refractive index $n_{g}$ and the analyser A. As previously, the plane of polarisation of the incident ray is controlled by the TN cell. The ray strikes the surface of the glass plate at the Brewster angle $\alpha_{B}=\arctan \left(n_{g}\right)$. If the cell is not subjected to external voltage, the electric field vector of the light wave is perpendicular to the plane of incidence and is partially reflected and partially transmitted after refraction by the angle $\beta$. After passing through the plate, the transmitted ray retains its initial direction but is eliminated by the analyser. The reflected beam goes out from the device. It is deviated from the initial direction by the angle $\delta=180^{\circ}-2 \alpha_{B}$ (Fig. 3a). Its intensity increases with the refractive index of glass. If the bias voltage unwinds the structure of the TN cell, then the electric field vector of the light wave is parallel to the plane of incidence. The reflection (a)

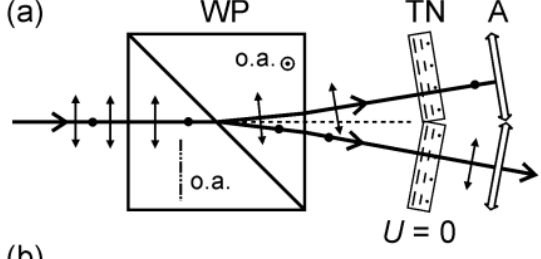

(b)

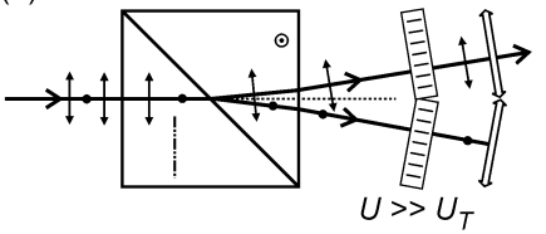

Fig. 2. Another variant of the device composed of twisted nematic cell, Wollaston prism and analyser A.

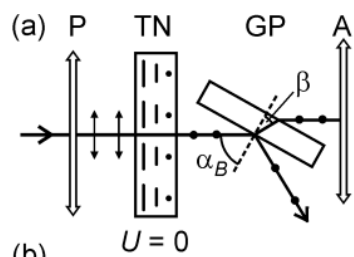

(b)

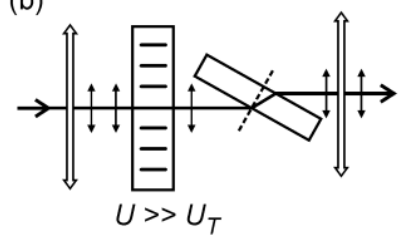

Fig. 3. Beam steering device using polarisation of light incident on a glass plate GP at Brewster's angle $\alpha_{B}$.

coefficient for this ray is zero. The beam is totally transmitted through the glass plate and propagates in the direction of the incident beam but is slightly shifted by the plate. The ray goes out from the system through the analyser as shown in Fig. 3b. In our example we adopted $n_{g}=1.778$ as for the heavy flint glass of type SF11 $(\lambda=632.8 \mathrm{~nm})[10]$. The corresponding Brewster angle is $\alpha_{B}=60.65^{\circ}$ and the deviation angle is $\delta=58.7^{\circ}$.

The next device proposed below is shown in Fig. 4. It is composed of entrance polariser $\mathrm{P}, \mathrm{TN}$ cell, rectangular reflecting prism RP made of heavy flint glass, calcite plate $\mathrm{CP}$ attached to the base of the prism and analyser A. The refractive index of glass, $n_{g}$, should be intermediate between $n_{e}$ and $n_{o}$ of calcite.

The incident beam is polarised by the entrance polariser and propagates parallel to the base of the prism. In the absence of voltage, the plane of polarisation is rotated in the TN cell, therefore the beam which enters the glass prism is polarised with the electric field vector perpendicular to the plane of incidence. It is refracted by the angle $\beta$ and reaches the boundary between glass and calcite at the angle $\phi=45^{\circ}+\beta$. This ray would propagate in the calcite plate as an extraordinary ray. 


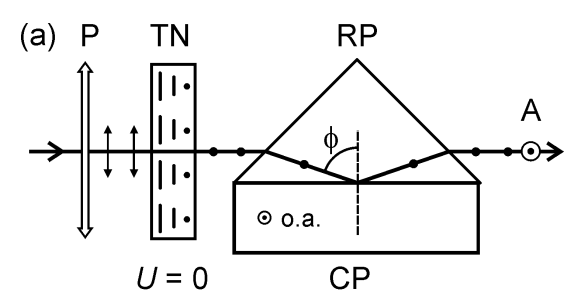

(b)

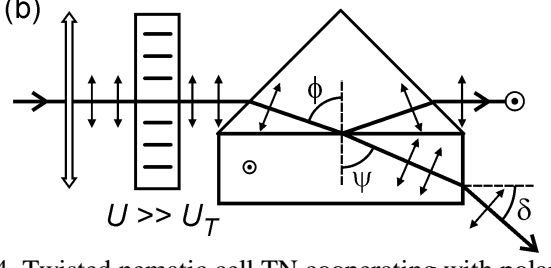

Fig. 4. Twisted nematic cell TN cooperating with polariser, totally reflecting prism $\mathrm{RP}$, calcite plate $\mathrm{CP}$ and analyser.

The refractive index of the calcite plate, $n_{e}$, is smaller than the refractive index of the glass, $n_{g}$, for this ray. Therefore total internal reflection occurs since the angle of incidence $\phi$ is larger than the critical angle $\phi_{c}=\arcsin \left(n_{e} / n_{g}\right)$. After refraction on the prism face it emerges from the prism along its initial direction and is transmitted through the analyser.

If the voltage of sufficient value is applied to the TN cell, then the electric field vector of light reaching the boundary between the glass prism and the calcite plate is perpendicular to the optic axis of the plate. The refractive index for this ray is $n_{o}$ which is larger than $n_{g}$. Therefore the light beam is partially transmitted to the calcite plate and partially reflected from the boundary. The reflected beam has very low intensity. It emerges from the prism and is eliminated by the analyser. In the presented example, the transmitted beam emerges the plate through its lateral surface, however the final direction of this beam can be different if the plane-parallel plate has another thickness or if it is replaced by calcite component of another shape. In our example we adopted $n_{g}=1.6051$ as for the heavy crown glass of type SK2 $(\lambda=632.8 \mathrm{~nm})$ [10]. The angle $\phi=71.13^{\circ}$ is larger than $\phi_{c}=67.71^{\circ}$ which ensures the total internal reflection of the ray in the absence of voltage.

The proposed beam steering devices are composed of the TN cell and commonly available optical components. They allow to change the direction of light beam by switching on and off the sufficiently high voltage applied to the cell. The speed of switching is limited only by the properties of the $\mathrm{TN}$ cell. The ideas of the proposed devices can be realised also in some alternative ways. For example, the Wollaston prism could be replaced by Rochon, Glan, Senarmont or Glan-Thompson prisms.

The TN cell was already applied in a beam deflecting device in which it cooperated with the polarising beam splitter PBS [11]. The device allowed to switch between the unchanged initial direction and the direction deflected by $90^{\circ}$. In Figure 5, another realisation of this concept is proposed, resulting in parallel shift of one of the rays. Both rays emerging from the PBS enter the same TN cell. If no voltage is applied, the analyser transmits one of the rays. After switching on the voltage, the other ray is transmitted.
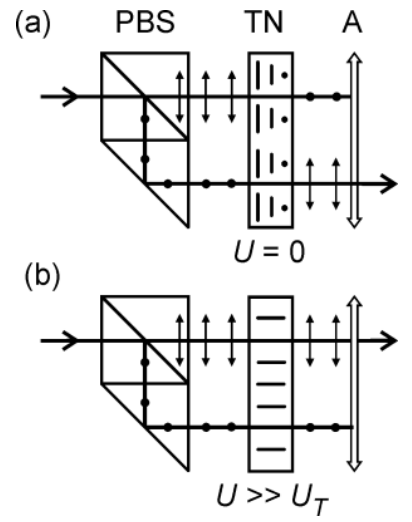

Fig. 5. Twisted nematic cell cooperating with polarising beam splitter PBS and analyser A

\section{References}

[1] P. F. McManamon, P.J. Bos, M.J. Escuti, J. Heikenfeld, S. Serati, H. Xie, E.A. Watson, Proc. IEEE 97, 1078 (2009).

[2] S.-T. Wu, D.-K. Yang, Fundamentals of Liquid Crystal Devices (John Wiley \& Sons, Chichester, 2006).

[3] X. Wang, D. Wilson, R. Muller, P. Maker, D. Psaltis, Appl. Opt. 39, 6545 (2000).

[4] T. Chan, E. Myslivets, J.E. Ford, Opt. Expr. 16, 14617 (2008).

[5] J. Kim, C. Oh, M.J. Escuti, L. Hosting, S. Serati, Proc. SPIE 7093, 709302 (2008)

[6] S. Valyukh, V. Chigrinow, H.S. Kwok, H. Arwin, Opt. Expr. 20, 15209 (2012).

[7] S. Valyukh, I. Valyukh, V. Chigrinov, Phot. Lett. Poland 3, 88 (2011).

[8] A.B. Golovin, S.V. Shiyanovskii, O.D. Lavrentovich, Proc. SPIE 5741, 146 (2005).

[9] M. Bass et. al., Handbook of Optics, vol. II $2^{\text {nd }}$ Ed., Ch. 8, (McGraw Hill, New York, 1995).

[10] Schott (Optical Glass Data Sheets, 2012). http://refractiveindex.info/download/data/2012/schott_optical_glass_col lection_datasheets_dec_2012_us.pdf

[11] R. Herke, M.H. Anderson, T. Baur, Liquid crystal in precision optical devices (Meadowlark Optics, Inc., 2005). 